
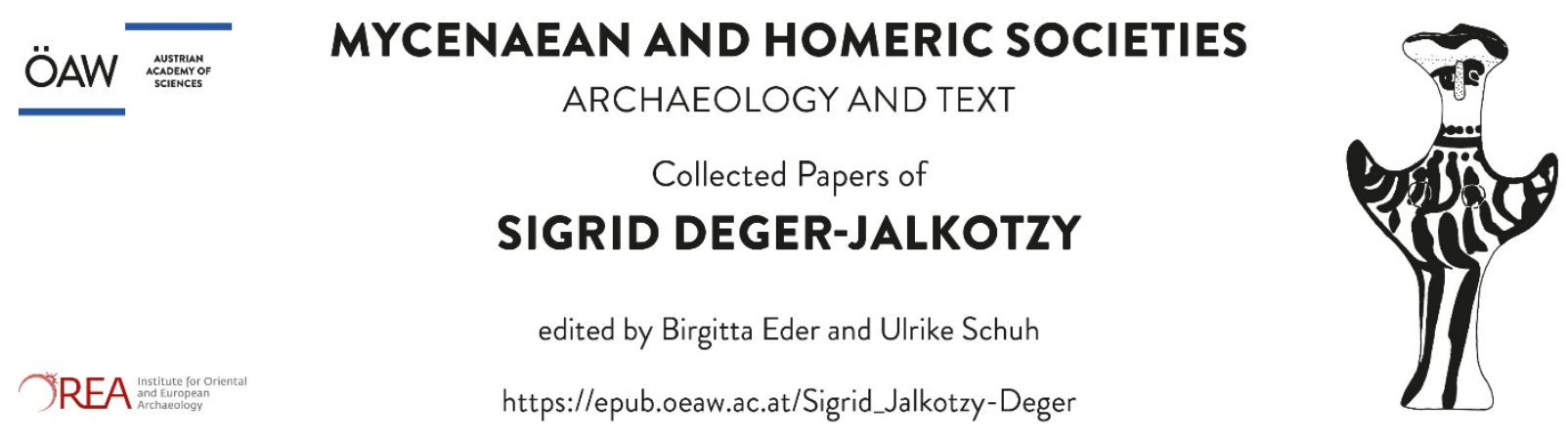

46.

S. Deger-Jalkotzy

\title{
Laudatio für Spyros Iakovidis
}

Almanach der Österreichischen Akademie der Wissenschaften 151, 2001, 136-137

(c) Verlag der ÖAW

mit freundlicher Genehmigung / with kind permission

Dieses Dokument darf ausschließlich für wissenschaftliche Zwecke genutzt werden (Lizenz CC BY-NC-ND), gewerbliche Nutzung wird urheberrechtlich verfolgt.

This document is for scientific use only (license CC BY-NC-ND), commercial use of copyrighted material will be prosecuted. 


\section{ÖSTERREICHISCHE \\ AKADEMIE DER WISSENSCHAFTEN}

\section{A L M A N A C H 2000/2001}

151. JAHRGANG

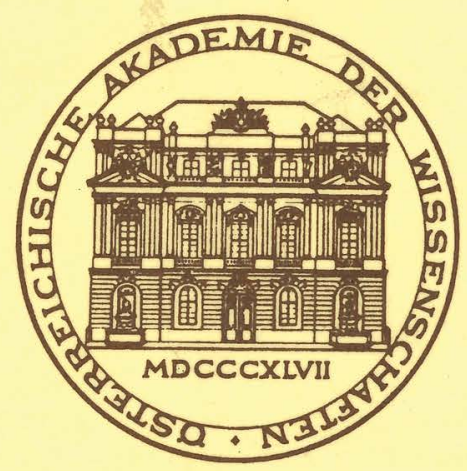

VERLAG DER

ÖSTERREICHISCHEN AKADEMIE DER WISSENSCHAFTEN WIEN 2001 


\author{
ÖSTERREICHISCHE \\ AKADEMIE DER WISSENSCHAFTEN
}

\title{
A L M A N A C H \\ $2000 / 2001$
}

151. JAHRGANG

WIEN 2001

VERLAG DER

ÖSTERREICHISCHEN AKADEMIE DER WISSENSCHAFTEN A-1010 WIEN, DR.-IGNAZ-SEIPEL-PLATZ 2 


\section{Präsidium}

\section{Präsident der Akademie}

und Vorsitzender der philosophisch-historischen Klasse

\section{Werner Welzig}

o. Professor der Neueren Deutschen Literaturgeschichte an der Universität Wien,

2345 Brunn am Gebirge, Enzersdorfer Straße 6, Tel. 022 36/342 69

\section{Vizepräsident der Akademie}

und Vorsitzender der mathematisch-naturwissenschaftlichen Klasse

Peter Schuster

o. Professor der Theoretischen Chemie an der Universität Wien,

1180 Wien, Dr.-Heinrich-Maier-Straße 60/12/10, Tel. 4402902

\section{Generalsekretär der Akademie}

und Sekretär der mathematisch-naturwissenschaftlichen Klasse

\section{Herbert Mang}

o. Professor der Elastizitäts- und Festigkeitslehre

an der Technischen Universität Wien,

1130 Wien, Tolstojgasse 5/10, Tel. 8776363

\section{Sekretär}

der philosophisch-historischen Klasse

\section{Herwig Friesinger}

o. Professor der Ur- und Frühgeschichte an der Universität Wien,

3424 Zeiselmauer, Bahnhofplatz 6, Tel. 022 42/312 95 


\section{Redaktioneller Hinweis}

Der vorliegende 151. Jahrgang umfaßt den Berichtszeitraum Oktober 2000 bis September 2001 bzw. Juni 2000 bis Mai 2001 für das Publikationsverzeichnis der Mitglieder

Redaktion: Johann Felfernig, Ingrid Weichselbaum

Alle Rechte vorbehalten

ISBN 3-7001-3035-X geb.,

ISBN 3-7001-3036-8 brosch.

ISSN $0378-8644$

Copyright (C) 2001 by

Österreichische Akademie der Wissenschaften

Wien

Druck: Grasl Druck \& Neue Medien, 2540 Bad Vöslau 


\section{Inhalt}

Präsidium

\section{Mitglieder}

Verzeichnis der Mitglieder der Österreichischen Akademie der Wissenschaften

Ehrenmitglied der Gesamtakademie

Mitglieder der mathematisch-naturwissenschaftlichen Klasse

Wirkliche Mitglieder . . . . . . . . . . . . . . . . 15

Ehrenmitglieder . . . . . . . . . . . . . . . . . 29

Korrespondierende Mitglieder im Inland . . . . . . . . . . 30

Korrespondierende Mitglieder im Ausland . . . . . . . . . 39

Mitglieder der philosophisch-historischen Klasse

Wirkliche Mitglieder . . . . . . . . . . . . . . . . 67

Ehrenmitglieder . . . . . . . . . . . . . . . . . 78

Korrespondierende Mitglieder im Inland . . . . . . . . . . 80

Korrespondierende Mitglieder im Ausland . . . . . . . . . 88

Veränderungen seit Gründung der Akademie . . . . . . . . . 111

Verstorbene Mitglieder der Akademie im Jahre 2000/2001

(Oktober-September) .

Neugewählte Mitglieder der Akademie im Jahre 2001

Mathematisch-naturwissenschaftliche Klasse .

Philosophisch-historische Klasse

\section{Publikationen}

Verzeichnis der im Jahre 2000/2001 (Juni-Mai) erschienenen Publikationen der wirklichen Mitglieder und der korrespondierenden Mitglieder im Inland

Mathematisch-naturwissenschaftliche Klasse

Philosophisch-historische Klasse 
Verzeichnis der im Jahre 2000/2001 (Oktober-September) erschienenen Publikationen der Österreichischen Akademie der Wissenschaften

Gesamtakademie

Mathematisch-naturwissenschaftliche Klasse

Philosophisch-historische Klasse 266

Die Feierliche Sitzung am 16. Mai 2001

Begrüßung durch den Präsidenten der Akademie Werner Welzig . . . 273

Bericht des Vizepräsidenten Peter Schuster . . . . . . . . . . . 277

Bericht des Generalsekretärs Herbert Mang . . . . . . . . . . . 281

Bericht des Sekretärs Herwig Friesinger . . . . . . . . . . . 291

Ansprache des Präsidenten . . . . . . . . . . . . . . . . 297

Festvortrag von w. M. Georg Stingl „Immunmanipulation - eine medizinische Herausforderung" . . . . . . . . . . . . . . 301

Verleihung der Akademie-Preise . . . . . . . . . . . . . 311

Präsidium und zentrale Verwaltung der Akademie

Präsidium

Präsidialsekretariat . . . . . . . . . . . . . . . . . 315

Rechts- und Personalangelegenheiten . . . . . . . . . . . 316

Verwaltungsstellen der beiden Klassen . . . . . . . . . . . . 316

Rechnungswesen und EDV-Belange . . . . . . . . . . . . . . 317

Bibliothek und Archiv . . . . . . . . . . . . . . . . . . . . . 317

Bauwesen . . . . . . . . . . . . . . . . . . . . . 318

Hausbetriebsstelle . . . . . . . . . . . . . . . . . . 318

Auslandsbeziehungen . . . . . . . . . . . . . . . . . . . . . 318

Stipendien- und Preisangelegenheiten . . . . . . . . . . . . . 319

Verlag . . . . . . . . . . . . . . . . . . . . . 319

Akademie-Rechenzentrum . . . . . . . . . . . . . . . 320

Betriebsrat . . . . . . . . . . . . . . . . . . . . 320

Sicherheitsvertrauenspersonen . . . . . . . . . . . . . . 321

Kommissionen der Akademie

Zusammensetzung und Mitarbeiter

Gemeinsame Kommissionen

Wissenschaftliche Kommissionen

Geschichte der Naturwissenschaften, Mathematik und Medizin . . . 323

Prähistorische K. . . . . . . . . . . . . . . . . . 324 
Quartärforschung

$\mathrm{K}$. für die wissenschaftliche Zusammenarbeit mit Dienststellen des BM für Landesverteidigung . . . . . . . . . . . . 325

Historische Pressedokumentation . . . . . . . . . . . . . . 325

Interdisziplinäre ökologische Studien . . . . . . . . . . . 326

Verwaltungskommissionen

Planungsk. . . . . . . . . . . . . . . . . . . . 326

Akademiebibliothek . . . . . . . . . . . . . . . . . . . 327

Finanzk. . . . . . . . . . . . . . . . . . . . . 327

Rechnungskontrollk. . . . . . . . . . . . . . . . . 327

Verwaltungsk. für die Stiftungen und Widmungen . . . . . . . 328

EDV-K. . . . . . . . . . . . . . . . . . . . . 328

Verlags- und Druckschriftenk. . . . . . . . . . . . . . . 328

Kommissionen der math.-nat. Klasse

Wissenschaftliche Kommissionen

Astronomie . . . . . . . . . . . . . . . . . . 330

Reinhaltung der Luft . . . . . . . . . . . . . . . . 330

Geophysikalische Forschungen . . . . . . . . . . . . . 331

Grundlagen der Mineralrohstofforschung . . . . . . . . . . . 331

Paläontologische und stratigraphische Erforschung Österreichs . . . 331

Wissenschaftliche Visualisierung . . . . . . . . . . . . 332

Verwaltungskommission

Budgetk. . . . . . . . . . . . . . . . . . . . . 332

Verleihung des Erwin-Schrödinger-Preises . . . . . . . . . 333

Verleihung des Felix-Kuschenitz-Preises . . . . . . . . . . 333

Verleihung des Erich-Schmid-Preises . . . . . . . . . . . . 333

Verleihung des Erich-Thenius-Stipendiums . . . . . . . . . 334

Verleihung des Alois-Sonnleitner-Preises . . . . . . . . . . . 334

Kommissionen der phil.-hist. Klasse

Kultur und Geschichte Österreichs und des Donauraumes

Historische K. . . . . . . . . . . . . . . . . . . . . . 335

Geschichte der Habsburgermonarchie . . . . . . . . . . . 335 
Schrift- und Buchwesen des Mittelalters . . . . . . . . . . 336

Literaturwissenschaft . . . . . . . . . . . . . . . . 337

Literarische Gebrauchsformen . . . . . . . . . . . . . 337

Kulturwissenschaften und Theatergeschichte . . . . . . . . 338

Rechtsgeschichte Österreichs . . . . . . . . . . . . . . . . 339

Kultur und Sozialgeschichte des römischen und byzantinischen

Reiches, des Mittelmeerraumes und des Nahen Ostens

Antike Rechtsgeschichte . . . . . . . . . . . . . . . . . . . . 339

Byzantinistik . . . . . . . . . . . . . . . . . . 339

Tabula Imperii Byzantini . . . . . . . . . . . . . . . . . 340

Numismatische K. . . . . . . . . . . . . . . . . . . . . . . . 341

Ägyptische K. . . . . . . . . . . . . . . . . . . . . . . 341

Mykenische K. . . . . . . . . . . . . . . . . . . 342

Balkan-K. . . . . . . . . . . . . . . . . . . 342

Kleinasiatische K. . . . . . . . . . . . . . . . . . . . 343

Allgemeine und indogermanische Sprachwissenschaft

Iranistik . . . . . . . . . . . . . . . . . . . . 344

Linguistik und Kommunikationsforschung . . . . . . . . . . . 344

Musik- und Kunstforschung

Kunstgeschichte .

Musikforschung.

Lexikographie und Editionen

Herausgabe des Corpus der lateinischen Kirchenväter (CSEL) . . . 346

Antike Literatur und lateinische Tradition . . . . . . . . . 346

Sozialanthropologie . . . . . . . . . . . . . . . . . . 347

Verwaltungskommissionen

Budgetk. . . . . . . . . . . . . . . . . . . . . 348

Verleihung des Wilhelm-Hartel-Preises . . . . . . . . . . . 348

Verleihung des Figdor-Preises . . . . . . . . . . . . . . 348

Moritz-Csáky-Widmung . . . . . . . . . . . . . . . . . . 349

Dr.-Friedrich-Teßmann-Sammlung . . . . . . . . . . . . . . 349

Unternehmungen der phil.-hist. Klasse . . . . . . . . . . . . . 349 
Institute und Forschungsstellen der Akademie Zusammensetzung der Kuratorien und Beiräte, Mitarbeiter

Institute der Gesamtakademie

Konrad-Lorenz-I. für Vergleichende Verhaltensforschung . . . . 351

Technikfolgen-Abschätzung . . . . . . . . . . . . . . 352

Phonogrammarchiv. . . . . . . . . . . . . . . . . 353

Österreichisches Biographisches Lexikon und biographische

Dokumentation . . . . . . . . . . . . . . . . . 354

Schallforschung . . . . . . . . . . . . . . . . . . 355

Institute der math.-nat. Klasse

Mittelenergiephysik . . . . . . . . . . . . . . . . 356

Hochenergiephysik . . . . . . . . . . . . . . . . . 356

Molekularbiologie . . . . . . . . . . . . . . . . . 360

Limnologie . . . . . . . . . . . . . . . . . . . 362

Diskrete Mathematik . . . . . . . . . . . . . . . . 364

Erich-Schmid-I. für Materialwissenschaft . . . . . . . . . . 365

Weltraumforschung . . . . . . . . . . . . . . . . 366

Biophysik und Röntgenstrukturforschung . . . . . . . . . . . 369

Biomedizinische Alternsforschung . . . . . . . . . . . . . . 370

Molekulare und Zelluläre Bioinformatik . . . . . . . . . . 372

IMP-IMBA Genome Research Center . . . . . . . . . . . 372

Molekulare Medizin . . . . . . . . . . . . . . . . 372

Zell- und Entwicklungsbiologie . . . . . . . . . . . . . . 373

Institute der phil.-hist. Klasse

Realienkunde des Mittelalters und der frühen Neuzeit . . . . . . . 373

Demographie . . . . . . . . . . . . . . . . . . 374

Stadt- und Regionalforschung . . . . . . . . . . . . . . 375

Kultur- und Geistesgeschichte Asiens . . . . . . . . . . . . . 376

Österreichische Dialekt- und Namenlexika . . . . . . . . . . 377

Kulturgeschichte der Antike . . . . . . . . . . . . . . . . . 379

Forschungsstellen

Geschichte des Mittelalters . . . . . . . . . . . . . 380

Institutioneller Wandel und europäische Integration . . . . . . . 381 
Forschungsprogramme

Man and Biosphere (MAB) . . . . . . . . . . . . . . . . . . 383

Hydrologie Österreichs . . . . . . . . . . . . . . . . . . . . 383

Internationales Geologisches Korrelationsprogramm (IGCP) . . . . 384

Global Change Program . . . . . . . . . . . . . . . . 385

International Decade for Natural Disaster Reduction (IDNDR) . . . 385

Geophysik der Erdkruste . . . . . . . . . . . . . . . 386

Alpenforschung . . . . . . . . . . . . . . . . . . . . . 386

Werkstoffkompetenzzentrum - Leoben Forschungsges.m.b.H. . . . . 387

Stipendienprogramme

Doktorandenprogramm . . . . . . . . . . . . . . . . 388

APART - Austrian Programme for Advanced Research and Technology 388

Max-Kade-Stipendien . . . . . . . . . . . . . . . . 333

Einrichtungen bei der ÖAW

Österreichische IIASA-K.

Kernfusionsforschung . . . . . . . . . . . . . . . . . . . . 391

Entwicklungsfragen . . . . . . . . . . . . . . . . . . . 393

Rekombinante Gen-Technik . . . . . . . . . . . . . . . . . 394

Kuratorium der Akademie für das Historische Institut beim

Österreichischen Kulturforum in Rom . . . . . . . . . . 394

Forschungszentrum: Diskurs, Politik, Identität . . . . . . . . . 395

Spezialforschungsbereich SCIEM 2000 . . . . . . . . . . . . . 395

In Verbindung mit der ÖAW

Ständiges Sekretariat der International Federation of Automatic

Control (IFAC) . . . . . . . . . . . . . . . . . . . . 397

Int. Lehrgang in Limnologie für Postgraduierte aus Entwicklungsländern 397

Arbeitsgemeinschaft für Kartographische Ortsnamenkunde (AKO) . . 397

\section{Delegationen}

Delegationen im Rahmen des ICSU. . . . . . . . . . . . . . . 398

Sonstige Delegationen . . . . . . . . . . . . . . . . . . . 403

Liste der von der Akademie zu vergebenden Preise . . . . . . . . . 411 
Gesellschaft der Freunde der ÖAW

Übersicht der Sitzungen der Akademie im Jahre 2001

\section{Nachrufe}

Auf Mitglieder der math.-nat. Klasse

\begin{tabular}{|c|c|c|c|c|c|c|c|c|c|}
\hline v. & & & & & & & & & \\
\hline EM & Karl Fellinger . . & & & & & & & & \\
\hline & Heinz Maier-Leibnitz & & & & & & & & \\
\hline & Günter Victor Schulz & & & & & & & & \\
\hline & iiszyn . & & & & & & & & \\
\hline & ber & & & & & & & & \\
\hline & & & & & & & & & \\
\hline & minger & & & & & & & & \\
\hline & & & & & & & & & \\
\hline & & & & & & & & & \\
\hline & Herbert Döring & & & & & & & & \\
\hline
\end{tabular}

Auf Mitglieder der phil.-hist. Klasse

w. M. Hans Thieme . . . . . . . . . . . . . . . . . . 499

w. M. Ulrich Schulz-Buschhaus . . . . . . . . . . . . 507

w. M. Erich Heintel . . . . . . . . . . . . . . . . 515

w. M. Ignaz Seidl-Hohenveldern . . . . . . . . . . . . . . 539

k. M. Dmitrij Sergeevič Lichačev . . . . . . . . . . . . 543

k. M. Hajime Nakamura . . . . . . . . . . . . . . . . . 553

k. M. Johannes Karayannopulos . . . . . . . . . . . . . 559

k. M. Dietrich W. H. Schwarz . . . . . . . . . . . . . 569

k. M. Josef Polišenský . . . . . . . . . . . . . . . . 573

k. M. Franciszek Sławski . . . . . . . . . . . . . . 581

Personenregister . . . . . . . . . . . . . . . . . . 589 


\section{Ehrenmitglied}

Iakovidis, Spyros: Geboren am 28. Februar 1923 in Athen. 1940

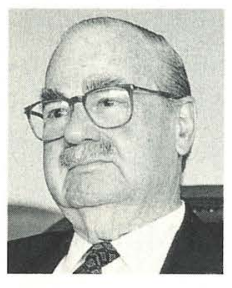

bis 1946 Studium der Altertumswissenschaften an der Universität Athen, mit klassischer, prähistorischer und byzantinischer Archäologie als Hauptfach. 1952-1954 Tätigkeit als Epimelet für die Altertümer beim staatlichen griechischen Antikendienst, betraut mit der Teilnahme an der Neuaufstellung des Akropolismuseums und mit der Aufsicht über alle Ausgrabungsarbeiten im Stadtbereich Athen. Daneben Beginn der Ausgrabungen in Perati (Attika). 1962 Promotion an der Universität Athen. Von 1970 bis 1974 war Spyros Iakovidis o. Professor für Archäologie an der Universität Athen. Anschließend kamen die Jahre seiner Lehrtätigkeit im Ausland. Nach den Gastprofessuren 1976 an der Universität Marburg und 1977 an der Universität Heidelberg wurde er 1978 als Professor für Klassische Archäologie an die Universität von Pennsylvania berufen, wo er bis 1991 wirkte. Gleichzeitig war er Curator des renommierten University Museums dieser Universität. Neben der Professur in den USA führte Iakovidis in seiner Heimat die Ausgrabungen in Gla durch. Nach der Pensionierung kehrte er nach Athen zurück, wo er die Direktion des Forschungszentrums für Altertumswissenschaften an der Akademie der Wissenschaften von Athen und die Leitung der griechischen Ausgrabungen in Mykene übernahm. Spyros Iakovidis steht in der Reihe jener großen griechischen Archäologen, die mit ihrem Lebenswerk die Grundlagen für die Erforschung der mykenischen Kultur Griechenlands geschaffen haben. Bleibendes Verdienst und weltweite Anerkennung über die Fachgrenzen der Mykenologie hinaus erwarb er sich durch seine 1953-1963 durchgeführten Ausgrabungen in der spätmykenischen Felskammergrab-Nekropole von Perati in Attika und die bewundernswert rasche Gesamtpublikation ihrer Ergebnisse in drei Bänden (1969-1970). Auch heute noch kann keine Studie zu den Folgen des Zusammenbruches der mykenischen Hochkultur, 
zur Chronologie und Sachkultur des frühen „Dunklen Zeitalters“ des 12. und 11. Jahrhunderts v. Chr., zur Frage von kultureller Kontinuität und Diskontinuität zwischen der mykenischen Epoche und der klassischen Antike Griechenlands auf dieses grundlegende und monumentale Werk verzichten. Einen bleibenden Platz nicht nur in den Bibliographien, sondern auch in der Wissenschaftsgeschichte, sicherte sich Spyros Iakovidis weiters durch die ebenfalls bereits vollständig publizierten Ergebnisse seiner Ausgrabungen in der palastzeitlichen mykenischen Großanlage von Gla in der böotischen Kopais, und ebenso durch seine bis heute nicht überholte Analyse mykenischer Wehrbauten. Hunderte von Vortragseinladungen an mehr als 80 Universitäten in Europa und Übersee zeugen von der Wertschätzung, die seine Leistungen gefunden haben. Weit davon entfernt, sich zur Ruhe zu setzen, leitet er an der Akademie von Athen das altertumswissenschaftliche Forschungszentrum und in Mykene die Ausgrabungen der Griechischen Archäologischen Gesellschaft. Seine Publikationstätigkeit reicht bis in die Gegenwart. Mit den Vertretern der mykenischen Forschung in Österreich steht Spyros Iakovidis seit den Tagen Fritz Schachermeyrs in freundschaftlicher Beziehung. In letzter Zeit durften sich vor allem die Mykenische Kommission der Österreichischen Akademie der Wissenschaften und der Spezialforschungsbereich SCIEM 2000 seiner tatkräftigen Unterstützung bei ihren Tätigkeiten in Griechenland und seiner Mitwirkung bei wissenschaftlichen Veranstaltungen erfreuen. Spyros Iakovidis ist Mitglied der Akademie der Wissenschaften in Athen und der Accademia Nazionale dei Lincei in Rom sowie Mitglied der großen archäologischen Institute und Gesellschaften Europas. Griechenland ehrte ihn mit der Würde eines Komturs des „Phönix-Ordens“. 